\title{
Optimum placement of PMU for wide area measurement systems
}

\author{
Mohan L. Kolhe*, a , N.P. Patidar ${ }^{\mathrm{b}}$, Laxmikant Nagar ${ }^{\mathrm{c}}$, Akshay Sharma ${ }^{\mathrm{d}}$, Vikash \\ K. Singh ${ }^{\mathrm{e}}$ \\ ${ }^{a}$ Faculty of Engineering \& Science, University of Agder, PO Box 422, NO 4604, Kristiansand, Norway \\ ${ }^{b}$ M.A. National Institute of Technology, Bhopal, India \\ ${ }^{c}$ University Institute of Technology, RGPV, Bhopal, India \\ ${ }^{d}$ Power Grid Corporation of India Ltd., Betul, India \\ ${ }^{e}$ Faculty of Computronics, I.G. National Tribal University, Amarkantak, 484 886, India
}

\begin{abstract}
:
This paper presents identification of a best selection process for selecting the most effective stabilizing signal to improve damping of inter area oscillations in a multimachine power system. Phasor Measurement Units or PMUs are a key element of the Wide Area Measurement Systems (WAMS). The large amount of data collected by PMUs need to be channelized to regional and global data centers where real-time state estimation, and protection, stabilization decisions are made. It is shown in this paper that how different signal selection techniques provides different control loop for damping of a particular inter area mode of oscillations. In spite of these the controller locations were obtained for optimum placement of phasor measurement units for wide area measurement systems. It is resulted from the two area 4 machine system that the HSV based signal selection approach performs excellent in both small and large disturbance for the test system compared to both residue and geometric measure of joint controllability and observability approach. Non-linear simulations are carried out in order to evaluate the performance of different approaches of signal selection under study.
\end{abstract}

Keywords: Inter-area oscillations, residue approach, geometric measures, power system stabilizer (PSS), Phasor Measurement Unit (PMU)

\section{Introduction}

Power system stability is one of the most crucial issues in power systems which deals with the response of the system to the disturbances occurred in the network. Some of the major oscillations attributed to system collapse are local mode, intra-plant mode, inter-area mode, control mode and torsional mode of oscillations [1]. From all these modes inter area mode of oscillations are very severe and observed over a large region of the network $[2,3]$. To make the system stable, it is very necessary that the inter-area modes of oscillations are effectively damped.

The conventional approach to damp inter area oscillation is to provide the supplementary control signal to the excitation system by installing Power System Stabilizer (PSS) at the generator location [4]. The PSS use the local stabilizing signal such as deviation of generator speed and provides the supplementary control input to the excitation. But the PSS taking local signal may not be always effective to damp inter area oscillations, because the local controllers are having lack of global observations [4,5]. It is also observed that the local signals have lack modal controllability and observability. But it is proved that under certain operating condition the inter area mode may be controllable from one area and observable from another control area. In this case the local controllers cannot provide effective damping.

In recent years, the global observation of the power system is achieved by using wide area

\footnotetext{
* Manuscript received March 1, 2018; revised July 3, 2018.

Corresponding author. Tel.: +47 3723 3293; E-mail address: mohan.1.kolhe@uia.no.

doi: $10.12720 /$ sgce.7.3.159-169
} 
measurement systems through phasor measurement units (PMUs) that receive signals from the satellite based global positioning system (GPS). The application of remote signal for damping controller has become successful due to the development of Phasor Measurement Units (PMUs) [6]. PMUs have very useful contribution in newly developed Wide Area Measurement System (WAMS) technology. The Phasor Measurement Unit is the central component of the WAMS that allows the measurement of the electric quantities of interest (voltage and current phasors, frequency and rate of change of frequency). PMUs also permits the synchronization and the transmission of the performed measurements.

PMUs can deliver synchronous phasors and control signals at high speed. PMUs are located remote to the controllers and the signals from PMU are referred as remote stabilizing signals. PMUs are positioned at strategic locations on the grid at the buses [7] and obtain a coherent picture of the entire network in real time. It is important to know that the phasor technology was not developed to replace the SCADA system, but to complement it.

Table 1. SCADA Vs PMU

\begin{tabular}{lll}
\hline \multicolumn{1}{c}{ Attribute } & \multicolumn{1}{c}{ SCADA } & PMU \\
\hline Measurement & Analog & Digital \\
Resolution & 2-4 Samples per second & up to 60 samples per seconds \\
Observability & Steady State & Dynamic/ Transient \\
Monitoring & Local & Wide Area \\
Phase Angle Measurement & No & Yes \\
Measured Quantity & Magnitude - (RMS) - MW, & Magnitude (RMS) and phase offset from common \\
& MVAR & reference - MW, MVAR, and Angle Difference
\end{tabular}

\section{Electromechanical Modes:}

\subsection{Intra plant mode}

Machines on the same power generation site oscillate against each other at 2.0 to $3.0 \mathrm{~Hz}$ depending on the unit ratings and the reactance connecting them.

\subsection{Local plant mode}

In local mode, one generator swings against the rest of the system at 1.0 to $2.0 \mathrm{~Hz}$. The impact of the oscillation is localized to the generator and the line connecting it to the grid.

\subsection{Inter-area mode oscillations}

This phenomenon is observed over a large part of the network. It involves two coherent group groups of generators swinging against each other at $1 \mathrm{~Hz}$ or less. The oscillation frequency is approximately 0.3 $\mathrm{Hz}$.

\subsection{Control mode oscillations}

These are associated with generators and poorly tuned exciters, governors, HVDC converters and SVC controls. Loads and excitation systems can interact through control modes.

\subsection{Torsional mode oscillations}

These modes are associated with a turbine generator shaft system in the frequency range of 10-46 Hz. Usually these modes are excited when a multi-stage turbine generator is connected to the grid system through a series compensated line. 


\section{Synchro-phasor}

A phasor is a complex number that represents both the magnitude and phase angle of voltage and current sinusoidal waveforms at a specific point in time (Fig. 1). Synchrophasor are precise timesynchronized measurements of certain parameters on the power system, now obtainable from grid monitoring devices called phasor measurement units (PMUs).

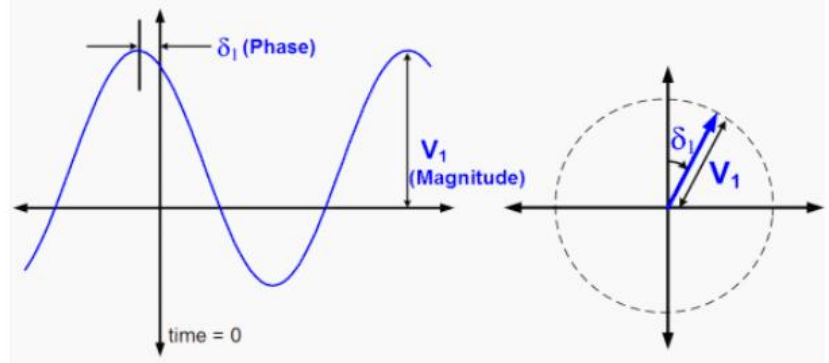

Fig.1. Sinusoidal waveform and phasor representation.

PMUs measure voltage, current and frequency and calculate phasors, and this aggregation of time synchronized grid condition data is called phasor data. Each phasor measurement is time stamped against Global Positioning System universal time; when a phasor measurement is time stamped, it is called a Synchrophasor [8,9]. This allows measurements taken by PMUs in different locations or by different owners to be synchronized and time-aligned, then immixed to provide a precise, comprehensive view of an entire region or interconnection [10-12]. PMUs sample at speeds of 30 observations per second, compared to conventional monitoring technologies (such as SCADA) that measure once every two to four seconds.

\section{Synchro-phasor Data Systems}

The purpose of a Synchrophasor data system is to make rapid measurements (at least 30 per second) of voltage and current phasors (magnitude and their respective phasor angles) that include precise time stamps and to make these measurements available to analyze and display grid conditions in transmission and power system control centers. Ultimately, Synchrophasor data systems may be used for automated control of the grid. A Synchrophasor system will meliorate real-time situational awareness and decision support tools to enhance system reliability. Synchrophasor measurements can also be used to improve component and system models for both on-line and off-line network analysis to assess system security and adequacy to withstand expected contingencies. The components of a Synchrophasor data system include:

\subsection{Phasor Measurement Unit (PMU)}

Calculates voltage and current phasors based on digital sampling of alternating current (AC) waveforms and an accurate time signal provided by a GPS clock. A PMU provides output data in a standard protocol at rates of at least 30 samples per second for communication to remote locations.

\subsection{Communication System}

A mechanism to transport the digital information from the PMU to the location where the data will be used. Communication is typically provided through a private wide-area network (WAN) but can be any digital transport system that offers acceptable security and availability; functional necessities for a Synchrophasor system architecture. 


\subsection{Phasor Data Concentrator (PDC)}

Receives and time-synchronizes phasor data from multiple PMUs to produce a real-time, time-aligned output data stream. A PDC can exchange phasor data with PDCs at other positions. Through use of multiple PDCs, multiple layers of concentration can be carried out within an individual Synchrophasor data system. A phasor data concentrator collects phasor data from multiple PMUs or other PDCs, aligns the data by time-tag to create a time-synchronized dataset, and passes this dataset on to other information systems. The functions of a PDC can vary depending on its role or its location between the source PMUs and the higher-level applications. A local PDC may be located physically close to PMUs (typically at a substation) to manage the collection and communication of time-synchronized data from local PMUs, send it to higher level concentrators, and store the data for use within the substation.

\subsection{Data Storage}

Systems to store Synchrophasor data and make it available in a convenient manner for post-real-time analysis can be integrated with the PDC or be stand-alone data historians or, in the case of smaller implementations, be traditional relational data base systems. Traditional relational databases can be used to manage phasor data for a short period of history or for implementations that contain a small number of PMUs [20]. Distributed non-relational databases are used to manage big datasets by large internet firms. These systems have some relational database features that can be used effectively with data systems that contain many, many terabytes of information. They divide the data up into smaller blocks that are processed in parallel. This technology is being explored for use in Synchrophasor data systems as well.

\section{Eigen Value Analysis}

For analyzing the small signal stability of any system, the system model can be linearized around an operating point i.e. the disturbances are considered to be so small or incremental in nature so that a linear model of the system around an operating point can be developed [13-14]. The state space representation of the power system can be written as

$$
\begin{gathered}
\dot{x}=A x+B u \\
y=C x
\end{gathered}
$$

Once the state space model of a power system is obtained the small signal stability of the system can be calculated and analysed and the eigenvalues $\lambda_{\mathrm{i}}$ are calculated for the A matrix. They are the non-trivial solutions of the equation:

$$
\operatorname{det}(\mathrm{A}-\lambda \mathrm{I})=0
$$

The solutions of characteristic equation are the eigenvalues of the $n \times n$ matrix $A$. These eigenvalues are of the form $\sigma \pm j \omega$. The stability of the operating point may be analyzed by studying the eigenvalues.

The conjugate-pair complex eigenvalues $(\sigma \pm j \omega)$ each corresponds to an oscillatory mode. A pair with a positive $\sigma$ represents an unstable oscillatory mode since these eigenvalues yield an unstable time response of the system. However, a pair with a negative $\sigma$ represents a desired stable oscillatory mode. Eigenvalues associated with an unstable or poorly damped oscillatory mode are also called dominant modes since their contribution dominates the time response of the system. It is quite obvious that the desired state of the system is for all of the eigenvalues to be in the left-hand side of the complex plane.

The damped frequency of the oscillation in Hertz and damping ratio are given by:

$$
\begin{gathered}
f=\frac{\omega}{2 \pi} \\
\xi=\frac{-\sigma}{\sqrt{\sigma^{2}+\omega^{2}}}
\end{gathered}
$$

The operating point is stable if all of the eigenvalues are on the left-hand side of the imaginary axis of 
the complex plane; otherwise it is unstable.

\section{Signal Selection And Controller Locations}

In order to damp the inter area oscillations; wide area controller employing signals from remote locations are very much necessary because they are more controllable and observable compared to local signals $[15,16]$. The remote stabilizing signals are often considered to as "global signals". In the selection of stabilizing signals and control location the effort should be to use as few measurement and control device as possible to achieve acceptable damping effect $[17,18]$.

The controllability and observability methods are often used to select controller location and stabilizing signals [19-21]. There are three different approaches are used for measurement and control signal selection such as

\section{1 . Residue approach}

The transfer function of an interconnected power system associated with the state equations can be uttered by

$$
\mathbf{G}(\mathbf{s})=\mathbf{C}(\mathbf{s I}-\mathbf{A})^{-\mathbf{1}} \mathbf{B}=\sum_{\mathbf{i}=\mathbf{1}}^{\mathbf{n}} \frac{\mathbf{R}_{\mathbf{i}}}{\left(\mathbf{s}-\boldsymbol{\lambda}_{\mathbf{i}}\right)}
$$

where $\boldsymbol{R}_{\boldsymbol{i}}$ is known as residue matrix of size $\mathbf{q} \times \mathbf{p}$ associated with $\lambda_{\boldsymbol{i}}$

$$
\mathbf{R}_{\mathbf{i}}=\mathbf{C} \phi_{\mathbf{i}} \Psi_{\mathbf{i}} B
$$

For $\mathbf{j}=\mathbf{1}, \mathbf{2}, \ldots \ldots, \mathbf{q}$ and $\mathbf{k}=\mathbf{1}, \mathbf{2}, \ldots \ldots, \mathbf{p}$ the elements of the residue matrix $\boldsymbol{R}_{\boldsymbol{i}}$ can be expressed as

$$
\mathbf{R}_{\mathbf{i}}(\mathbf{j}, \mathbf{k})=\mathrm{C}_{\mathbf{j}} \phi_{\mathrm{i}} \Psi_{\mathbf{i}} \mathbf{B}_{\mathbf{k}}
$$

In fact the residue can be represented as the product of the mode's controllability and observability.

The controllability for the mode $\mathbf{i}$ at $\mathbf{k}^{\text {th }}$ generator can be represented as

$$
\operatorname{cont}_{\mathbf{j}, \mathrm{k}}=\left|\boldsymbol{\Psi}_{\mathbf{i}} \mathbf{B}_{\mathbf{k}}\right|
$$

The observability of the mode $\mathbf{i}$ from $\mathbf{j}^{\text {th }}$ output is defined by

$$
\mathbf{o b j}_{\mathbf{j}, \mathbf{k}}=\left|\mathbf{C}_{\mathbf{j}} \phi_{\mathbf{i}}\right|
$$

From above equations, it is concluded that

$$
\left|\mathbf{R}_{\mathbf{i}}\right|=\left|\mathbf{C}_{\mathbf{j}} \phi_{\mathbf{i}} \Psi_{\mathbf{i}} \mathbf{B}_{\mathbf{k}}\right|=\mathbf{o b j}_{\mathbf{j}, \mathbf{k}} * \operatorname{cont}_{\mathbf{j}, \mathbf{k}}
$$

It has been proved that the PSS is installed at that generator where largest residue for the $\mathbf{i}^{\text {th }}$ mode is found.

The limitation of the residue approach is that the approach is only valid for signals of the same type. However signals of widely varying physical significance such as tie-line power flow (MW), bus frequency $(\mathrm{Hz})$, shaft speed ( $\mathrm{rad} / \mathrm{s})$, angle shift (deg.), etc. will be involved in the output matrix simultaneously, then residue approach suffers a lot from scaling problem. To overcome this problem geometric approach is used.

\subsection{Geometric approach}

Optimal selection of measured signal and control site location is performed with the geometric measures of joint controllability and observability approach. The indices for controllability and observability $\mathrm{COI}_{\mathrm{k}}$ and $\mathrm{OBI}_{\mathrm{j}}$ for a particular $\boldsymbol{i}^{\text {th }}$ mode of oscillations are defined by:

$$
\operatorname{coI}_{k}=\cos \left(\theta\left(\Psi_{i}, B_{k}\right)\right)=\frac{\left|\Psi_{i_{i}} B_{k}\right|}{\left\|\Psi_{i}\right\|\left\|B_{k}\right\|}
$$




$$
\operatorname{OBI}_{\mathbf{j}}=\cos \left(\theta\left(\phi_{\mathrm{i}}, \mathrm{C}_{\mathrm{j}}\right)\right)=\frac{\left|\mathrm{C}_{\mathrm{j}} \phi_{\mathrm{i}}\right|}{\left\|\mathrm{C}_{\mathrm{j}}\right\|\left\|\phi_{\mathrm{i}}\right\|}
$$

where, $b_{k}$ is the $\mathbf{k}^{\text {th }}$ column of input matrix B (corresponding to the ith input), $\boldsymbol{\Psi}_{\mathbf{i}}$ is the left eigenvector of $\mathbf{i}^{\text {th }}$ mode of oscillation and $c_{j}$ is the $\mathbf{j}^{\text {th }}$ row of output matrix $C$ (corresponding to the $\mathbf{j}^{\text {th }}$ output). $\boldsymbol{\phi}_{\mathbf{i}}$ is right eigenvector of $\mathbf{i}^{\text {th }}$ mode of oscillations. The symbol | | and \| $\|$ are the absolute value of a scalar and standard- 2 norm respectively. The joint controllability and observability index of geometric approach is defined by

$$
\text { Joint-index } \mathrm{k}, \mathrm{j}=\mathbf{C O I}_{\mathbf{k}} * \mathbf{O B} \mathbf{I}_{\mathbf{j}}
$$

If $\mathrm{COI}_{\mathrm{k}}=0$, then the mode $\mathrm{k}$ is uncontrollable from input $\mathrm{i}$ and $\mathrm{If}_{\mathrm{OBI}}=0$, then the mode $\mathrm{k}$ is unobservable from the output $\mathrm{j}$.

\subsection{Hankel singular value based approach}

As in the above two cases HSV also can be used for the measure of controllability and observability index of the given system. For a linear system, the HSV can be defined as

$$
\sigma_{i}=\sqrt{\lambda_{i}(P Q)}, \quad i=1, \ldots, n
$$

Where $\sigma_{i}$ reflects the index of joint controllability and observability and $\sqrt{\lambda_{i}(P Q)}$ reflects the $i^{t h}$ eigenvalue of $\mathrm{PQ}$. The $\mathrm{P}$ and $\mathrm{Q}$ are controllability and observability Gramian matrix respectively. The (A, B) are controllable if the controllability Gramian P is defined as

$$
P=\int_{0}^{\infty} e^{A t} B B^{T} e^{A^{T} t} d t
$$

Matrix $\mathrm{P}$ is a solution of the following Lyapunov equation

$$
A P+P A^{T}+B B^{T}=0
$$

The $(\mathrm{A}, \mathrm{C})$ are observable if the observability Gramian matrix is defined as

$$
Q=\int_{0}^{\infty} e^{A^{T} t} C C^{T} e^{A t} d t
$$

Matrix $\mathrm{Q}$ is the solution of following Lyapunov equation:

$$
A^{T} Q+Q A+C^{T} C=0
$$

Now in the remaining sections the above methods of selection of control signal will be tested in the case study example.

\section{Test System}

Despite the high level of activity in recent years on wide-area control, there are many aspects remain poorly understood due to the complexity in large power system. Therefore a small Kundur's two area 4 machine power system has been set up to deal with the most of the issues/challenges faced by wide-area control. In the test system all the generators are equipped with governor, AVR, and IEEE ST1A type static exciter [13]. The loads taken here are constant impendence type and connected to bus no. 7 and 9. The structure of the case study power system is given in Fig.2 


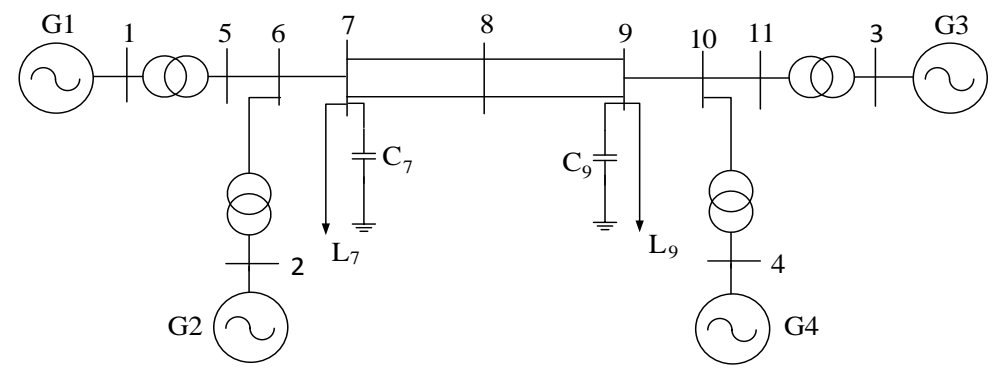

Fig. 2 Kundur's two area four machin system [1].

It is assumed that the Local signal based PSSs are assumed to be connected with the chosen generators that are determined as suitable control location, for damping of local area oscillations. After the calculation of most suitable stabilizing signal and control location a Global signal based PSS is employed supplement to the input of AVR along with Local PSS for the damping of inter area oscillations [16]. The exporting Power $\mathrm{P}_{\text {tie }}$ from area 1 to area 2 through tie line is $413 \mathrm{MW}$ and chosen as nominal operating point.

\section{Small Signal Stability Analysis}

Power system dynamic components of the studied system are modelled according to the Kundur's benchmark system database. With the detailed modal analysis, it came to know that each generator has 11 states and the total order of the nonlinear model is 44 . In normal operating condition, the power transfer through tie-line connecting two areas is $413 \mathrm{MW}$ and load consumption of area 1 is $967 \mathrm{MW}$ and of area 2 is $1767 \mathrm{MW}$. The nonlinear model of the studied system is linearized around the initial operating point of tie-line power $\left(\boldsymbol{P}_{\text {tie }}=\mathbf{4 1 3} \mathbf{M W}\right)$. After performing modal analysis with Power System Toolbox (PST), 44 modes with their eigenvalues, damping ratio and frequency are obtained which are tabulated in Table 2.

Table 2. Two area system modes of oscillations

\begin{tabular}{cccc}
\hline Mode No. & Eigen Value & Damping Ratio & Frequency $(\mathrm{Hz})$ \\
\hline 5 & $-0.2522-0.6536 \mathrm{i}$ & 0.3601 & 0.1040 \\
15 & $0.0453-4.1135 \mathrm{i}$ & -0.011 & 0.6547 \\
\hline
\end{tabular}

There is only one inter-area mode having frequency of $0.6547 \mathrm{~Hz}$, presented in the system. This mode is referred to lightly damp inter-area mode 15 with eigenvalue $(0.0453-4.1135 i)$ and damping ratio of 0.011 . The objective of the proposed WADC is aimed at achieving adequate acceptable damping for this mode.

In addition to the critical mode identification, modal analysis was also performed for coherent machine identification where the one group of generators forms one area and oscillating with another group of generators [17]. Compass plot shows the coherent areas in the studied system as shown in Fig. 3. 


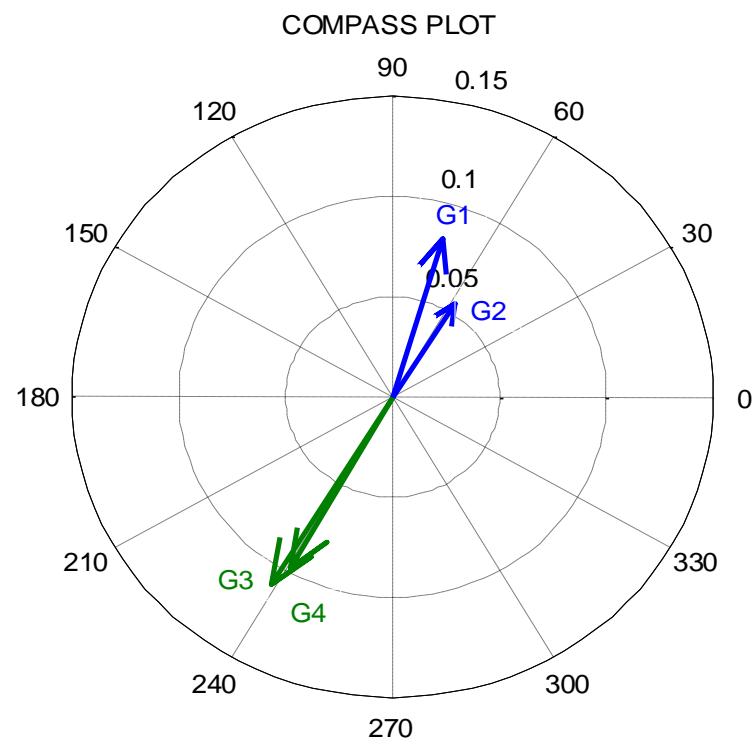

Fig. 3. Coherent Area Identification using Compass Plot.

There are four arrows representing the four generators. The length and direction of each arrow is corresponding to the magnitude and phase angle of respective eigenvector for the critical mode of machine. In the compass plot, it is clear that generator 1 and generator 2 forms area 1, and generator 3 and generator 4 forms another area so that the studied system termed as two area system when area 1 is oscillating with respect to area 2 . The small signal stability analysis [18] is performed under no fault condition and by applying small disturbance of 0.05 to the voltage reference of exciter in generator 1 of area 1.

\section{Optimal Signal Selection and Control Site Location}

The tie-line active power flow at various lines of the system has been taken for signal selection. The signal selection is carried out by residue approach, geometric measure of controllability/observability approach and Hankel Singular Value (HSV) based signal selection approach. Table 3, Table 4 and Table 5 show results obtained from Residue approach, Geometric approach and HSV approach respectively.

Table 3. Normalized values of the Residue approach for the 4 machine two area system

\begin{tabular}{ccccc}
\hline \multirow{2}{*}{ Signals } & \multicolumn{5}{c}{ Generators } \\
\cline { 2 - 5 } & G-1 & G-2 & G-3 & G-4 \\
\hline P6-7 & 0.5298 & 0.6849 & 0.5206 & 0.6686 \\
P7-8 & 0.3545 & 0.4584 & 0.3484 & 0.4475 \\
P8-9 & 0.3224 & 0.4169 & 0.3168 & 0.4070 \\
P9-10 & 0.7735 & 1 & 0.7601 & 0.9763 \\
\hline
\end{tabular}


Table 4. Normalized values of the geometric measure of controllability/observability approach for 4 machine 11 bus system.

\begin{tabular}{ccccc}
\hline & \multicolumn{4}{c}{ Signals } \\
\cline { 2 - 5 } Generators & $\boldsymbol{P}_{\mathbf{6 - 7}}$ & $\boldsymbol{P}_{\mathbf{7 - 8}}$ & $\boldsymbol{P}_{\mathbf{8}-\mathbf{9}}$ & $\boldsymbol{P}_{\mathbf{9 - 1 0}}$ \\
\cline { 2 - 5 } & 0.3528 & 0.7735 & 0.7655 & 0.4179 \\
G-1 & 0.4560 & 1 & 0.9897 & 0.5402 \\
G-2 & 0.3466 & 0.7601 & 0.7522 & 0.4106 \\
G-3 & 0.4452 & 0.9763 & 0.9662 & 0.5274 \\
\hline
\end{tabular}

Table 5. Absolute values of HSV for 2 area 4 machine system

\begin{tabular}{ccccc}
\hline \multirow{2}{*}{ Generators } & \multicolumn{4}{c}{ Signals } \\
\cline { 2 - 5 } & $\boldsymbol{P}_{\mathbf{6 - 7}}$ & $\boldsymbol{P}_{\mathbf{7 - 8}}$ & $\boldsymbol{P}_{\mathbf{8 - 9}}$ & $\boldsymbol{P}_{\mathbf{9 - 1 0}}$ \\
\hline G-1 & $\mathbf{8 . 6 0 3 0 \boldsymbol { e } ^ { - \mathbf { 0 4 } }}$ & 0.0178 & 0.0111 & 0.0274 \\
G-2 & 0.0052 & 0.0860 & 0.1075 & 0.3661 \\
G-3 & 0.1192 & 0.4895 & 0.3264 & 0.8804 \\
G-4 & 0.1878 & 0.4361 & 0.3304 & 1 \\
\hline
\end{tabular}

It can be observed from above results that the signal selected by HSV based signal selection approach provides better response as compared to residue geometric approach (Fig. 4). It is also resulted that in case of large disturbance, the HSV based signal selection approach performs excellent in comparison with residue and geometric approach (Fig. 5).

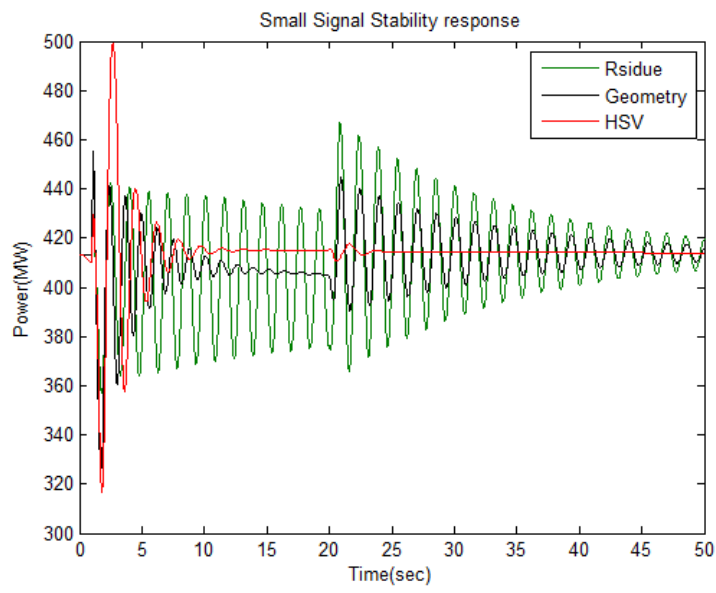

Fig. 4 Tie line active power flow due to step change in voltage reference at generator-1. 


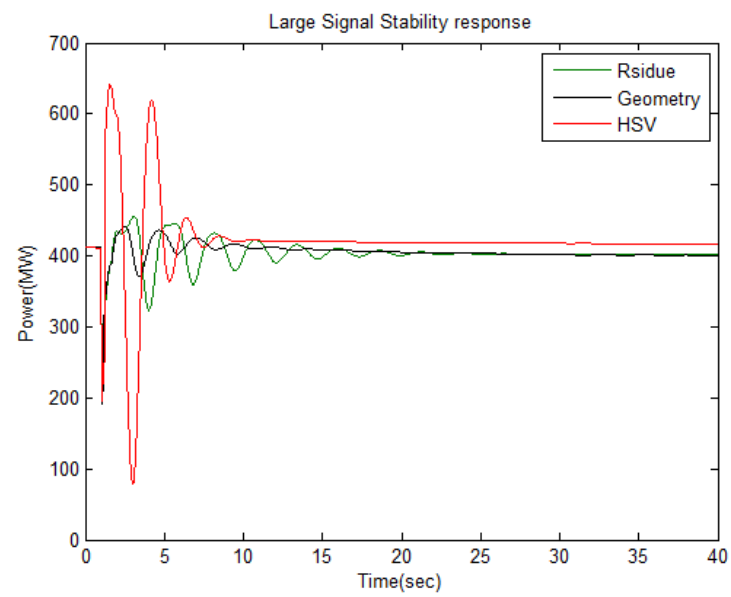

Fig. 5 Tie line active power response for 3 phase fault at bus 8 .

\section{Conclusion}

The selection of control loop for a given inter area mode by three different method of signal selection approaches are obtained. In order to validate the result of three different methods, the Kundur's two area four machine system has been used for the case study example. The responses of Tie line active power flow is investigated for three methods i.e. Residue, Geometrical and HSV are observed. The controller locations for the optimum placement of PMU for wide area measurement system are obtained.

\section{Acknowledgement}

The University of Agder (Norway) and I.G. National Tribal University (India) gratefully acknowledge the partial support for this work of the project 'India Norway Cooperation Program Project INCP 2014/10110', which is jointly funded by the University Grants Commission (India) and the Norwegian Centre for International Cooperation in Education.

\section{References}

[1] Kundur P, Paserba J, Ajjarapu V, Andersson G, Bose A, Canizares C, Hatziargyriou N, Hill D, Stankovic A, Taylor C., Van Cutsem T, Vittal V. Definition and classification of power system stability IEEE/CIGRE joint task force on stability terms and definitions. IEEE Transactions on Power Systems, 19(3):1387-1401, Aug. 2004

[2] Padhy B, Srivastava SC, Verma NK, A Coherency Based Approach for Signal Selection for Wide Area Stabilizing Control in Power Systems. IEEE Sys. J., 7(4): 807-816, Dec 2013.

[3] Kundur P. Power System Stability and Control. New York, NY, USA: McGraw-Hill, 1994

[4] P. Bikash, Choudhury, 'Robust Control in Power Systems', Springer, 2005.

[5] Erlich I, Hashmani A, Shewarega F. Selective damping of inter area oscillations using Phasor Measurement Unit (PMU) signals, Proceeding of IEEE conference Power Tech, Pub: IEEE, Trondheim, 2011: 1-6.

[6] Heniche A, Kamwa I. Assessment of two methods to select wide-area signals for power system damping control. IEEE Transc. Power Syst. 23(2): 572-581 May 2008.

[7] Andrea C, Nicola L, Carlo M. A flexible GPS-Based system for synchronized phasor measurement in electric distribution networks. IEEE Transactions on Instrumentation and Measurement, 57(11) November 2008

[8] Chi-Shan Y, Chih-Wen L. A new PMU-based fault location algorithm for series compensated lines. IEEE Transactions On Power Delivery, 17(1) January 2002.

[9] Ming Z, Virgilio A. C, James ST, Arun GP. An alternative for including phasor measurements in state estimators. IEEE Transactions on Power Systems, 21(4), November 2006.

[10] Ela A, Sallam AJ. McCalley, Fouad A. Damping controller design for power system oscillations using global signals. IEEE Trans. Power Syst., 11(2): 767-773, May 1996.

[11] Almutairi AM. Optimal input and output signal selection for wide-area controllers. IEEE Bucharest Power Tech Conference, Bucharest, Romania, Pub: IEEE, 2009, June 28th -July 2nd, pp. 1-6. 
[12] Anaparthi K, Chaudhuri B, Thornhill N, Pal B. Coherency identification in power systems through principal component analysis. IEEE Trans. Power Syst., 20(3): 1658-1660, Aug. 2005.

[13] Patidar NP, Kolhe ML, Tripathy NP, Sahu B, Sharma A, Nagar LK, Azmi AN. "Optimized design of wide-area PSS for damping of inter-area oscillations. in Proc. 2015 IEEE 11th International Conference on Power Electronics and Drive Systems (IEEE PEDS) 2015, Sydney, Australia, 1172-1177, June 2015.

[14] Akshay S, Nagar LK, Sahu B, Tripathy NP, Patidar NP. "Time latency compensation for wide area damping controller. 6th IEEE Power India International Conference, DTU, Delhi, December 2014.

[15] David ML, Robert JB, Paul B, Iyad AK, Luigi V, John MD. The Open PMU Platform for Open-Source Phasor Measurements. IEEE Transactions on Instrumentation and Measurement. 62(4), April 2013.

[16] Jin M, Pu Z, Hong-jun F, Bo B, and Zhao-yang D. Application of phasor measurement unit on locating disturbance source for low-frequency oscillation. IEEE Transactions on Smart Grid, 1(3), December 2010.

[17] Patidar NP, Kolhe ML, Sharma A, Nagar LK, Tripathy NP, Sahu B. Optimal signal selection of wide area damping controller considering time delay in multi-machine power system. 2015 IEEE PES Asia-Pacific Power and Energy Engineering Conference, Brisbane, Australia, 1-5, Nov, 2015.

[18] Kolhe M, Patidar NP, Nagar LK, Sharma A. Coherency identification and oscillation damping effects of PSS in interconnected power system: A comparative analysis approach. 2016 IEEE/IAS 52nd Industrial and Commercial Power Systems Technical Conference (I\&CPS), Detroit, MI, 2016, pp. 1-6.

[19] Andrea C, Nicola L, Carlo M, Sara S. A flexible GPS-based system for synchronized phasor measurement in electric distribution networks. IEEE Transactions on Instrumentation and Measurement, 57(11), November 2008.

[20] Jie Y, Chen-Ching L, Umesh V. PMU-Based monitoring of rotor angle dynamics. IEEE Transactions on Power Systems. 26(40, November 2011

[21] Mai RK, He ZY, Fu L, He W, Bo ZQ. Dynamic phasor and frequency estimator for phasor measurement units, IET Generation, Transmission \& Distribution, 2010, 4(1): 73 -83. 